\title{
EDITORIAL
}

\section{CT FFR A paradigm shift in evaluation of coronary artery disease}

\author{
Anirudh Kohli
}

Breach Candy Hospital Trust Mumbai, Maharashtra, India. E-mail: dranirudhkohli@gmail.com

Invasive catheter coronary angiography has long been considered the reference standard for diagnosis of coronary artery disease. Decisions regarding revascularization procedures have traditionally been based on visual assessment of severity of coronary artery disease, often termed occulo-stenotic reflex. $70 \%$ is the cutoff to decide on revascularization. However, there are numerous limitations of visual interpretation, significant inter and intra observer variability as well as factors other than luminal diameter narrowing, that influence the physiologic significance of a focal narrowing. Whether the narrowing is focal or diffuse, single or multiple, lesion length, shape and eccentricity as well as presence of collaterals. The decision to revascularize coronary artery stenosis should be governed by hemodynamic significance of a lesion rather than visual angiographic severity.

Fractional flow reserve (FFR) is an invasive technique performed at the time of cardiac catheterization to determine the maximal achievable blood flow in the presence of a stenosis versus maximal flow in the absence of stenosis. Essentially to determine how much blood flow at maximal hyperemia is being reduced by the lesion thus determining the hemodynamic significance of the lesion. To determine FFR, pressure at the aortic level as well as distal to the lesion at maximal hyperemia is taken during cardiac catheterization. This ratio provides the FFR value. A value below 0.8 is considered to be hemodynamically significant.

The FAME ${ }^{[1]}$ trial looked at two groups - one group received percutaneous coronary intervention solely on the basis of visual stenosis on angiography, and the second received percutaneous coronary intervention based on a FFR below 0.8 . The primary end point for the study was death, myocardial infarction and repeat revascularization. The purpose of any therapy is to reduce the incidence of all these three end points. In the FAME trial, there was a $72 \%$ reduction in the primary endpoint in the second group (FFR group). The long-term efficacy of this study was confirmed in 2 and 5 year follow-ups. This has led to a paradigm shift towards using functional/hemodynamic significance of a coronary lesion (FFR) rather than anatomic severity of coronary stenosis. In current practice based on visual assessment, all stenosis above $70 \%$ are revasularized. An interesting observation of the FAME trial was 20\% of lesions above $70 \%$ had a FFR above 0.8 thus received intervention with no benefit. The reason being FFR takes into account ante grade as well as collateral flow, but visual assessment takes into account only ante grade flow. Chronically infarcted myocardium may have a visually significant stenosis. Vascularizing this will be off no value. Chronically infarcted myocardium has a low metabolic requirement. Therefore, the flow will not be reduced and FFR will not be reduced. In the FAME study, more importantly $35 \%$ of lesions with a visual assessment between $50-70 \%$ had a FFR below 0.8 and would benefit from intervention. This is as long/tandem or proximal lesions may cause compromise in supply to large parts of myocardium, though the stenosis may not be angiographically significant, the FFR will be low. Therefore, FFR is of paramount importance to decide on revascularization especially for stenosis between $50-80 \%$.

CT coronary Anglo over the last decade has evolved into a premier tool for noninvasive evaluation of coronary arteries. It has excellent negative predictive value. Thus, its ability to rule out coronary artery disease is excellent. Its positive predictable value is moderate as it tends to overestimate stenosis, nearly $50 \%$ of significant lesions on CT do not have flow limiting disease on invasive FFR. Further, it provides only anatomic information and no functional information. To obtain functional information noninvasive myocardial perfusion studies need to be performed. All these studies stress the heart. Single photon emission CT (SPECT), CT perfusion, MR perfusion studies may be performed. SPECT is the most popular comprising of more than $90 \%$ of all noninvasive myocardial perfusion test performed. It has its share of limitations, there is a significant radiation burden due to the radiopharmaceutical used. Another significant limitation is it diagnostic capabilities are based on relative distribution of radiopharmaceuticals in the myocardium. This is qualitative/subjective evaluation. If there is a balanced distribution between the coronary artery territories as happens in triple vessel disease the study will be falsely negative. Other noninvasive tests such as CT perfusion and MR perfusion have a slightly higher accuracy than SPECT, but are unfortunately not widely used due to their limited availability, limited expertise and cost.

The ideal would be to have a single study providing anatomical and functional information in a single 
noninvasive test. Since CT Anglo fulfils the anatomic requirements, it is only logical to utilize its capabilities further.

CT FFR has been developed which applies. Computational fluid dynamic techniques to the CT Anglo data. This estimates FFR values throughout the coronary artery tree. Since virtual hyperemia is built into the model there is no need to stress the heart or use adenosine. This is ideal as in one test without stressing the heart. Numerous studies such as DISCOVER- FLOW, ${ }^{[2]}$ Follow up-NXT ${ }^{[3]}$ have compared the efficacy of CT FFR to invasive FFR with excellent correlation. In fact, CT FFR reclassified $68 \%$ of false positives on CTA as true negatives thus sorting out the limitation of CTA of moderate predictive value. There was a reduction of $61 \%$ catheter angiograms in patients with $>50 \%$ stenosis based on CTFFR.

Another significant limitation of CT Anglo are calcific plaques. These tend to bloom on CT Anglo thus obscuring

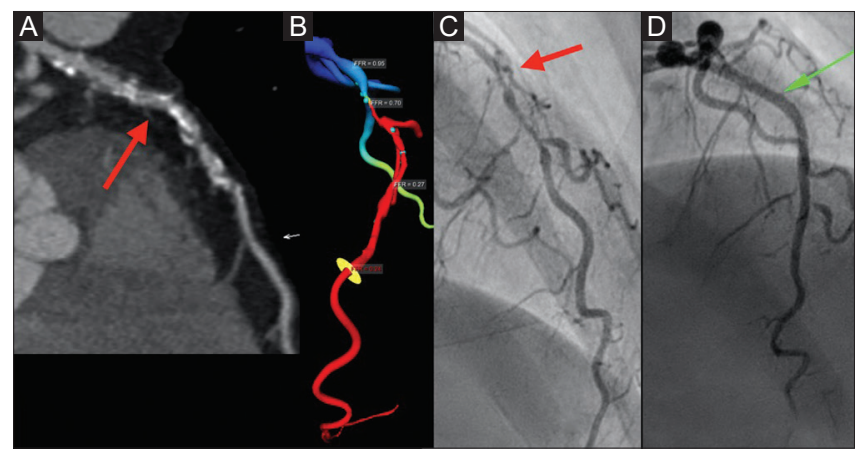

Figure 1 (A-D): (A) CT Angio demonstrates diffuse disease with soft and calcific plaques in proximal LAD. (B) CT FFR demonstrates low FFR 0.70 (red). (C) Invasive catheter angio confirms marked narrowing (D) Post angioplasty angiogram shows excellent result

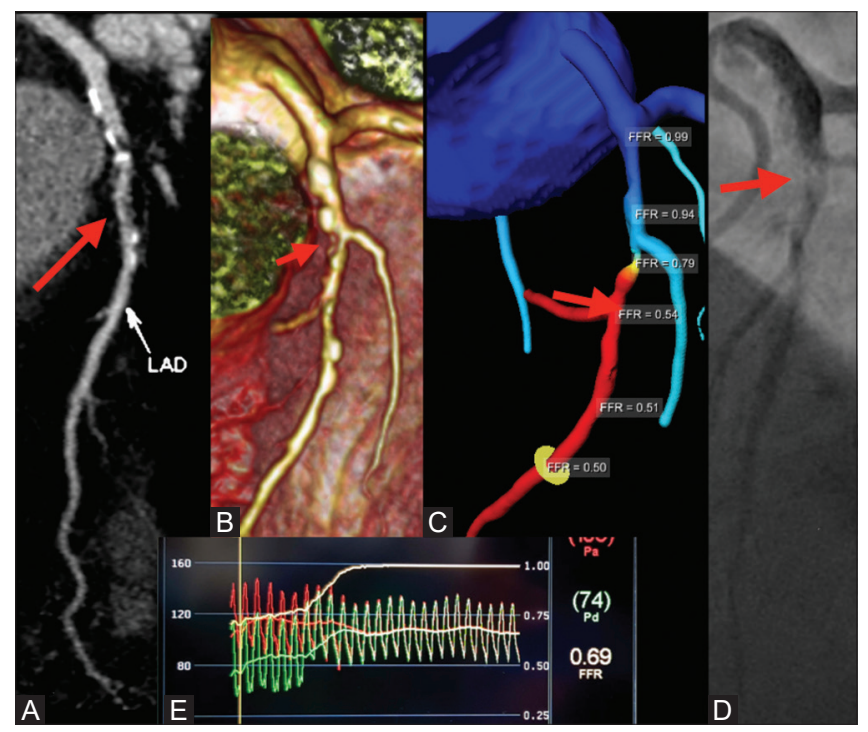

Figure 3 (A-E): (A) CT Angio shows significant disease in proximal LAD, confirmed on VRT (B) CT FFR (C), invasive angio (D), Invasive FFR (E) the lumen, these segments of the coronary artery are not readable. Obtaining information regarding these segments is possible only by doing additional tests such as myocardial perfusion or invasive catheter angiograms. Fortunately, FFR is not affected by calcium, the FFR at these unreadable segments can be determined so additional tests are not required.

Though CT FFR [Figures 1-4] was FDA approved in 2014, it is unfortunately not widely used as this requires a supercomputer for the computation of CTFFR. CT Anglo data has to be sent to an offsite computer for analysis, resulting in a turnaround time which can range from 24 hours to a week. Being a propriety software the cost of data analysis is high ranging from INR 75,000 to 1.25 lacs

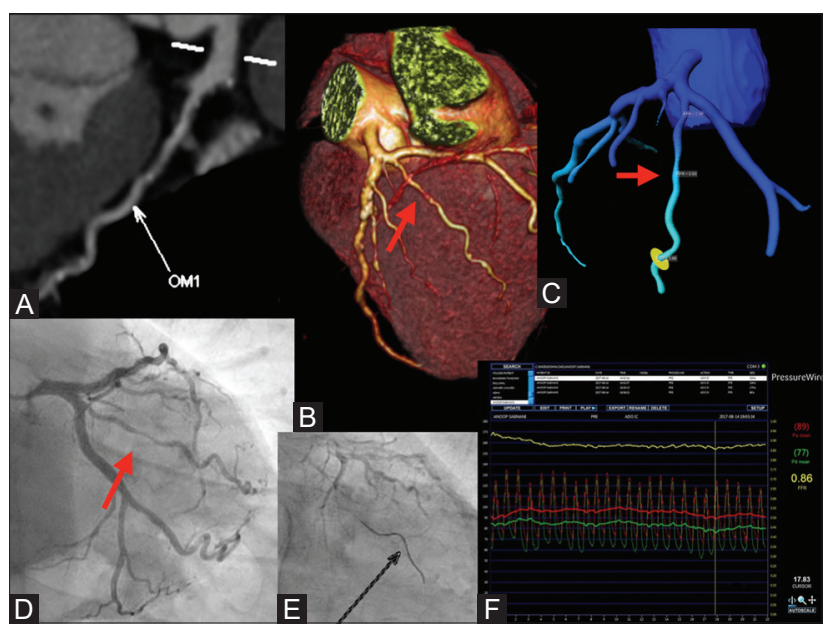

Figure 2 (A-F): (A) CT Angio reveals narrowing in OM1, (B) VRT also demonstrates narrowing, (C) CT FFR reveals narrowing not functionally significant as FFR above 0.8, (D) Catheter angiogram demonstrates narrowing in OM1, (E) Pressure wire beyond narrowing to measure distal pressure, (F) Invasive FFR confirms CT FFR finding of 0.86

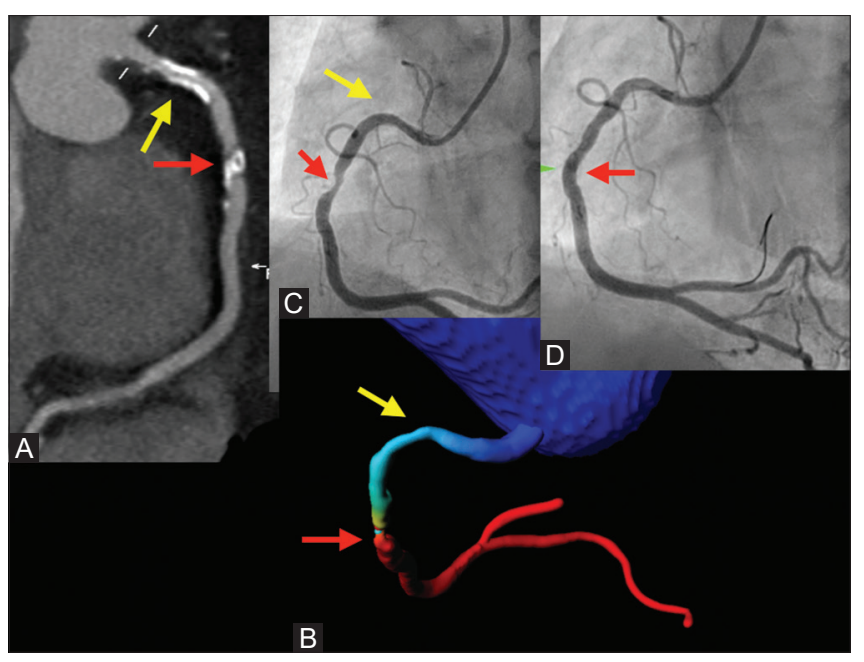

Figure 4 (A-D): (A) CT Angio reveals calcific plaques in proximal RCA narrowing lumen (yellow arrow), Dense calcific plaques in mid RCA obscuring visualisation of lumen (red arrow), (B) CT FFR reveals functional significance of dense plaque, confirmed on invasive cath angio (C) Post angioplasty image (D) 
per case! Fortunately, a newer CTFFR technique has been introduced based on deep machine learning using artificial intelligence to compute functional severity of a lesion. In this new model computation time is reduced by 80 -fold thus allowing analysis utilizing on site workstations with nearly real time analysis. Numerous studies ${ }^{[4-6]}$ have compared this new technique with invasive FFR finding it to be accurate. Another useful utility is Virtual stenting, to predict FFR following stenting, how much would flow increase and if any possible residual ischemia.

A combination of CT Anglo and CT FFR provides a mine of information regarding coronary artery disease. With excellent anatomy on CTA, the extent of coronary artery disease is demonstrated. The extent of luminal compromise, focal or diffuse, single or multiple plaques, tended lesions. The morphology of these plaques can be assessed for high risk features. The addition of CT FFR provides information regarding the impact of plaque on blood flow helping to decide further clinical management, especially avoiding unnecessary invasive testing or to guide appropriate revascularization. In fact, the combination of CT Anglo and CTFFR will become the gatekeeper to the invasive cardiac catheterization lab, deciding who needs intervention and who needs medical therapy/lifestyle management or nothing at all!!

\section{References}

1. Tonino PA, Fearon WF, De Bruyne B, Oldroyd KG, Leesar MA, Ver Lee PN, et al. Angiographic versus func- tional severity of coronary artery stenoses in the fame study fractional flow reserve versus angiography in multivessel evaluation. J Am Coll Cardiol 2010;55:2816-21.

2. Koo BK, Erglis A, Doh JH, Daniels DV, Jegere S, Kim HS, et al. Diagnosis of ischemia-causing coronary stenoses by noninvasive fractional flow reserve computed from coronary computed tomographic angiograms. Results from the prospective multicenter discover-flow (diagnosis of ischemia-causing stenoses obtained via noninvasive fractional flow reserve) study. J Am Coll Cardiol 2011;58:1989-97.

3. Nørgaard BL, Leipsic J, Gaur S, Seneviratne S, Ko BS, Ito H, et al. Di- agnostic performance of noninvasive frac- tional flow reserve derived from coronary computed tomography angiography in sus- pected coronary artery disease: The NXT trial (analysis of coronary blood flow using CT Angiography: Next steps). J Am Coll Cardiol 2014;63:1145-55.

4. Yang DH, Kim YH, Roh JH, Kang JW, Ahn JM, Kweon J, et al. Diagnostic performance of on-site CT-derived fractional flow reserve versus CT perfusion. Eur Heart J Cardiovasc Imaging 2017;18:432-40.

5. De Geer J, Sandstedt M, Björkholm A, Alfredsson J, Janzon M, Engvall J, et al. Software-based on-site estimation of fractional flow reserve using standard coronary CT angiography data. Acta Radiol 2016;57:1186-92.

6. Kruk M, Wardziak Ł, Demkow M, Pleban W, Pręgowski J, Dzielińska Z, et al. Workstation-based calculation of CTA-based FFR for intermediate stenosis. JACC Cardiovasc Imaging 2016;9:690-9.

This is an open access journal, and articles are distributed under the terms of the Creative Commons Attribution-NonCommercial-ShareAlike 4.0 License, which allows others to remix, tweak, and build upon the work non-commercially, as long as appropriate credit is given and the new creations are licensed under the identical terms.

\begin{tabular}{|l|l|}
\hline \multicolumn{2}{|c|}{ Access this article online } \\
\hline Quick Response Code: & \\
\hline & Website: \\
\hline & www.ijri.org \\
\cline { 2 - 3 } & DOI: \\
\hline
\end{tabular}

Cite this article as: Kohli A. CT FFRA paradigm shift in evaluation of coronary artery disease. Indian J Radiol Imaging 2019;29:233-5.

Received: 09-Oct-2019

Accepted: 12-Oct-2019
Revision: 11-Oct-2019

Published: 30-Oct-2019 